\title{
Generating synthetic CT from low-dose cone-beam CT by using generative adversarial networks for adaptive radiotherapy
}

Liugang Gao ${ }^{1,2 \dagger}$, Kai Xie ${ }^{1,2+}$, Xiaojin Wu ${ }^{3}$, Zhengda Lu ${ }^{1,2,4}$, Chunying Li $\mathrm{Li}^{1,2}$, Jiawei Sun ${ }^{1,2}$, Tao Lin ${ }^{1,2}$, Jianfeng Sui ${ }^{1,2}$ and Xinye $\mathrm{Ni}^{1,2^{*}}$

\begin{abstract}
Objective: To develop high-quality synthetic $\mathrm{CT}$ ( $\mathrm{SCT}$ ) generation method from low-dose cone-beam $\mathrm{CT}$ (CBCT) images by using attention-guided generative adversarial networks (AGGAN) and apply these images to dose calculations in radiotherapy.

Methods: The CBCT/planning CT images of 170 patients undergoing thoracic radiotherapy were used for training and testing. The CBCT images were scanned under a fast protocol with 50\% less clinical projection frames compared with standard chest M20 protocol. Training with aligned paired images was performed using conditional adversarial networks (so-called pix2pix), and training with unpaired images was carried out with cycle-consistent adversarial networks (cycleGAN) and AGGAN, through which sCT images were generated. The image quality and Hounsfield unit $(\mathrm{HU})$ value of the $\mathrm{s} C \mathrm{~T}$ images generated by the three neural networks were compared. The treatment plan was designed on $\mathrm{CT}$ and copied to $\mathrm{SCT}$ images to calculated dose distribution.
\end{abstract}

Results: The image quality of SCT images by all the three methods are significantly improved compared with original CBCT images. The AGGAN achieves the best image quality in the testing patients with the smallest mean absolute error (MAE, $43.5 \pm 6.69$ ), largest structural similarity (SSIM, 93.7 \pm 3.88 ) and peak signal-to-noise ratio (PSNR, $29.5 \pm 2.36$ ). The $s C T$ images generated by all the three methods showed superior dose calculation accuracy with higher gamma passing rates compared with original CBCT image. The AGGAN offered the highest gamma passing rates $(91.4 \pm 3.26)$ under the strictest criteria of $1 \mathrm{~mm} / 1 \%$ compared with other methods. In the phantom study, the sCT images generated by AGGAN demonstrated the best image quality and the highest dose calculation accuracy.

Conclusions: High-quality sCT images were generated from low-dose thoracic CBCT images by using the proposed AGGAN through unpaired CBCT and CT images. The dose distribution could be calculated accurately based on SCT images in radiotherapy.

Keywords: Synthetic CT, Low-dose CBCT, Attention-guided GAN, Unpaired, Adaptive radiotherapy

*Correspondence: nxy@njmu.edu.cn

${ }^{\dagger}$ Gao Liugang and Xie Kai contributed equally to this work

${ }^{1}$ Radiotherapy Department, Second People's Hospital of Changzhou, Nanjing Medical University, Changzhou 213003, China

Full list of author information is available at the end of the article

\section{Introduction}

Cone-beam CT (CBCT) images are widely used in imageguided radiotherapy (IGRT) [1-3], and they are important for decreasing the positioning error and increasing the accuracy of treatments for patients with cancer. Compared with images from traditional fan-beam CT, CBCT images suffer from low contrast and artifacts due original author(s) and the source, provide a link to the Creative Commons licence, and indicate if changes were made. The images or other third party material in this article are included in the article's Creative Commons licence, unless indicated otherwise in a credit line to the material. If material is not included in the article's Creative Commons licence and your intended use is not permitted by statutory regulation or exceeds the permitted use, you will need to obtain permission directly from the copyright holder. To view a copy of this licence, visit http://creativecommons.org/licenses/by/4.0/. The Creative Commons Public Domain Dedication waiver (http://creativeco mmons.org/publicdomain/zero/1.0/) applies to the data made available in this article, unless otherwise stated in a credit line to the data. 
to X-ray scattering, mechanical accuracy, and movement of patients during scanning $[4,5]$, resulting in serious distortion of the Hounsfield unit (HU) value. Hence, CBCT images are unsuitable for calculating dose distributions for replanning in adaptive radiotherapy. Besides, patients may undergo multiple CBCT scans during an IGRT treatment course and this raises a great concern about delivered dose to the patients. Previous study indicated that daily CBCT scan for IGRT could increase the secondary cancer risk by $2 \%$ up to $4 \%$ [6]. To reduce the additional dose for patients generated from IGRT, the researchers have proposed several low-dose CBCT imaging technologies $[7,8]$. Now, the low-dose protocols of CBCT scanning have been widely used in clinical practice.

Many methods of using CBCT images in adaptive radiotherapy have been proposed, and these include water-air-bone density assignment $[9,10]$, CBCT imaging process improvement based on modeling [11-13], and deformable image registration (DIR) of CT/CBCT images $[14,15]$. Direct HU-ED calibration of CBCT images has relatively low accuracy due to the absence of artifact reduction processing. Electron density assignment is time consuming and influenced by human experience. Arai [16] modified the $\mathrm{HU}$ values of CBCT images to match the planning $\mathrm{CT}$ images by the histogram matching algorithm and evaluated in the phantom and head and neck cancer patients. Traditional modelbased CBCT imaging correction is often realized by creating complex physical models to simulate the scattering [17-20] or changing of hardware. This method is difficult to promote due to limitations in hardware or calculation efficiency of physical models. Mainegra-Hing [17] calculated the scatter contribution of CBCT in the phantom by Monte Carlo (MC) algorithm. Niu [19] proposed a priori CT-based scatter correction method, where the corresponding planning $\mathrm{CT}$ projections were used to correct CBCT projections, and evaluated using two phantom studies. Park [20] applied the priori CT-based scatter correction technique to phantoms and a prostate patient for proton dose calculation. The priori CT-based scatter correction method is on the premise that the anatomical structure of CBCT is completely consistent with that of planning $\mathrm{CT}$ after registration which is difficult to satisfied in clinical practice such as the thorax and abdomen. DIR transforms planed CT to CBCT through deformation registration to account for anatomical changes. This type of method achieves good results at sites that are stationary, such as the head and neck. However, the method's registration accuracy needs to be improved at sites with considerable anatomic structural changes, such as the chest and abdomen [15].

Another method of correcting the $\mathrm{HU}$ value of $\mathrm{CBCT}$ images is to generate synthetic CT (sCT) images from
CBCT images through deep learning [21-31]. This method establishes a complicated mapping between $\mathrm{CBCT}$ and $\mathrm{CT}$ by training neural networks, thus allowing $\mathrm{SCT}$ images to be generated from $\mathrm{CBCT}$ directly. sCT has the same anatomical structure as $\mathrm{CBCT}$, and the $\mathrm{HU}$ values of tissues are close to those of planning CT. Chen [22] used Unet to generate sCT images from the CBCT of patients with head and neck cancer, and the loss function combined the mean absolute error (MAE) and structural similarity index (SSIM). The MAE of sCT and $\mathrm{CT}$ in the testing results was $18.98 \mathrm{HU}$. Similarly, $\mathrm{Li}$ [21] added a residual unit to Unet to generate $\mathrm{SCT}$ from CBCT from patients with head and neck cancer, and the MAE between $\mathrm{sCT}$ and $\mathrm{CT}$ ranged within 6-27 HU. Instead of generating sCT directly, Hansen [28] proposed a ScatterNet where pairs of measured and corrected projections were trained using a Unet-like architecture. The corrected projection was obtained by the priori CT-based scatter correction method [19]. Lalonde [29] applied the $\mathrm{MC}$ simulation to generate $\mathrm{CBCT}$ projections for head and neck patients, then the Unet was trained to reproduce $\mathrm{MC}$ projection-based scatter correction from raw projections. The MAE of scatter-corrected images was 13.4 $\mathrm{HU}$, compared to $69.6 \mathrm{HU}$ for the uncorrected images. Landry [30] compared Unet training with three different datasets to correct $\mathrm{CBCT}$ images for prostate patients. The datasets include raw and corrected $\mathrm{CBCT}$ projections, raw $\mathrm{CBCT}$ image and DIR-synthetic CTs, raw $C B C T$ image and reconstructed $C B C T$ image based on corrected projections. Supervised learning methods, such as Unet [32], require paired CBCT/CT images as the training dataset, and voxel-wise loss is usually applied. However, these methods need high-accuracy alignment of paired images, which is difficult to acquire in clinics, especially at sites with considerable anatomical structural changes, such as the chest and abdomen.

The development of generative adversarial networks (GAN) [33] has provided a new technology and framework for the application of medical images. GAN has achieved state-of-the-art performance in many medical image tasks, including segmentation [34, 35], classification [36, 37] and medical image synthesis [38-40]. Isola[41] proposed conditional adversarial networks(cGAN) in image-to-image translation (so-called pix2pix) which was widely used in medical image reconstruction [40] and cross modality synthesis [38, 39]. Maspero [38] applied pix2pix in MR-tosCT generation on 2D paired transverse image slices of 32 prostate cancer patients. Cusumano [39] used cGAN to generate sCT from low field $\mathrm{MR}(0.35 \mathrm{~T})$ images in pelvis and abdomen for MR-guided adaptive radiotherapy. Quan [40] reconstructed the MR image form under-sampled K-space using pix2pix. 
Zhu [42] proposed an unsupervised cycle-consistent adversarial network (cycleGAN) to solve image translation for unpaired datasets, and it has been applied extensively in unpaired medical image translation [43]. Liang [23] utilized cycleGAN to generate sCT from the CBCT of patients with head and neck cancer by using an unpaired training dataset; a phantom experiment demonstrated that the method has better anatomical accuracy than the DIR method. Kida [26] conducted training on unpaired CBCT/CT images of 20 patients with prostate cancer by using cycleGAN and found that the image quality of $\mathrm{sCT}$ substantially improves compared with that of the original CBCT. Harms [24] fed 3D image patches to cycleGAN for CBCT-to-sCT image generation of patients with brain and pelvis cancer. They used paired CBCT and CT images in training and found that the mean absolute errors (MAEs) of sCT in the brain and pelvis are 13.0 and $16.1 \mathrm{HU}$, respectively. On the basis of the study of Harms [24], Liu [25] added self-attention to the generator network of cycleGAN for CBCT-to-sCT image generation of patients with pancreatic cancer and calculated the radiotherapy dose distribution. These studies on sCT generation from $\mathrm{CBCT}$ images concentrated on the head or abdomen, but limited studies have been conducted on CBCT images of the thorax, and lowdose CBCT-to-sCT image generation have not been studied.

In this study, unpaired low-dose $\mathrm{CBCT}$ and $\mathrm{CT}$ images of the thorax were trained using GAN. The lowdose $\mathrm{CBCT}$ images were obtained under a fast protocol with 50\% less clinical projection frames compared with standard protocol. The $\mathrm{sCT}$ images generated from $\mathrm{CBCT}$ were used to calculate the dose distribution for adaptive radiotherapy. Given that the anatomical structure changes considerably due to respiratory movement, acquiring perfect alignment for $\mathrm{CT} / \mathrm{CBCT}$ images is difficult. Hence, GAN was selected for unsupervised training. Furthermore, the low-dose CBCT images of the thorax include considerable artifacts, such as streaking, shading, and cupping caused by $\mathrm{X}$-ray scatter and respiratory movements of patients; these artifacts disturb image translation tasks. We used attention-guided GAN (AGGAN) [44], which pays attention to the important part of images to eliminate numerous artifacts. Moreover, cycleGAN [42] and conditional GAN (so-called pix2pix) [41] were used in CBCT-to-sCT generation, and the quality of $\mathrm{sCT}$ images generated by different neural networks was compared. Then, a quantitative assessment of the generated $\mathrm{sCT}$ images was performed on a thoracic phantom, and the dose distribution of a radiotherapy plan was calculated.

\section{Materials and methods}

\section{Image acquisition and processing}

The low-dose CBCT and planning CT images of 170 patients who underwent free-breathing thoracic radiotherapy in our hospital were collected, 136 pairs as the training dataset and 34 pairs as testing dataset. The CBCT images were acquired through XVI scanning of a linear accelerator Infinity (Elekta, Stockholm, Sweden). In this study, a fast $\mathrm{CBCT}$ protocol was used for scanning to obtain low-dose CBCT images. Compared with the built-in standard protocol, a fast protocol accelerates the gantry rotation speed and decreases the scanning frames, thus decreasing the scanning time and radiation dose of patients. However, image quality is reduced to some extent $[8,45]$. The fast protocol was realized by modifying the gantry rotation speed of the standard chest M20 protocol from $180^{\circ} / \mathrm{min}$ to $360^{\circ} / \mathrm{min}$ while the other parameters were kept constant. The projection frames were reduced from 660 to 330 for each patient scan. The gantry was rotated by $360^{\circ}$ during each CBCT scanning, and 330 projection frames were collected. The planning CT images of patients were acquired using Siemens CT (SOMATOM Force, Germany). The scanning and reconstruction parameters of $\mathrm{CBCT}$ and $\mathrm{CT}$ are listed in Table1. The CT images were re-sampled to keep their resolution consistent with that of the $\mathrm{CBCT}$ images. Then, the CBCT images of each patient were used as fixed images, and the corresponding CT images were aligned with the $\mathrm{CBCT}$ images via $3 \mathrm{D}$ rigid registration. For the testing dataset, a deformable registration was performed on the $\mathrm{CT}$ to pair it to the corresponding CBCT by a multi-resolution B-spline algorithm. Afterward, the $\mathrm{CT}$ images were cropped to have the same field of view (FOV) as the corresponding CBCT images.

\section{Image synthesis with AGGAN}

AGGAN has a similar network structure as cycleGAN [44], and it involves two generators $\left(G_{C B C T-C T}\right.$ generates CT from CBCT and $G_{C T-C B C T}$ generates CBCT from CT) and two discriminators $\left(D_{C T}\right.$ distinguishes the $\mathrm{SCT}$ from the real CT and $D_{C B C T}$ distinguishes the synthetic CBCT (sCBCT) from real CBCT). AGGAN is composed of two

Table 1 The scanning and reconstruction parameters of CBCT and planning CT image

\begin{tabular}{llllll}
\hline & $\begin{array}{l}\text { Tube } \\
\text { voltage } \\
(\mathbf{k V P})\end{array}$ & $\begin{array}{l}\text { Tube } \\
\text { current } \\
(\mathbf{m A})\end{array}$ & $\begin{array}{l}\text { Spatial } \\
\text { resolution } \\
\left(\mathbf{m m}^{\mathbf{2}}\right)\end{array}$ & $\begin{array}{l}\text { Slice } \\
\text { thickness } \\
\mathbf{( m m})\end{array}$ & $\begin{array}{l}\text { Image } \\
\text { size }\end{array}$ \\
\hline $\mathrm{CBCT}$ & 120 & 20 & $1 \times 1$ & 2 & $410 \times 410$ \\
$\begin{array}{l}\text { Planning } \\
\text { CT }\end{array}$ & 120 & 220 & $0.97 \times 0.97$ & 2.5 & $512 \times 512$ \\
\hline
\end{tabular}


cycles. In the first cycle, $\mathrm{CBCT}$ images are inputted into $G_{C B C T-C T}$ to generate sCT images. Then, sCT images are inputted into $G_{C T-C B C T}$ to generate recycled CBCT (rCBCT) images. The two discriminators distinguish the corresponding generative images. The cycle-consistency loss constrains the generation process to minimize the differences between the original $\mathrm{CBCT}$ images and the $\mathrm{rCBCT}$ images. In the second cycle, $\mathrm{CT}$ images are inputted into $G_{C T-C B C T}$ to generate $\mathrm{SCBCT}$ images, which are then fed into $G_{C B C T-C T}$ to generate recycled $C T$ ( $\mathrm{rCT}$ ) images. Compared with the original cycleGAN, AGGAN modifies the generator network, which is equipped with a built-in attention module. A cycle process of AGGAN is shown in Fig. 1. $G_{C B C T-C T}$ contains the encoding and decoding parts. The encoding part is a downsampling process that shares weights. The decoding part contains two branches; one generates $n-1$ content masks, and the other generates $\mathrm{n}$ attention masks. The attention masks are divided into $\mathrm{n}-1$ foreground attention masks and one background attention mask after applying the Soft$\max$ function. Soft $\max \left(A_{i}\right)=e^{A_{i}} / \sum_{c=1}^{n} e^{A_{c}}$, in which
$A$ is attention masks and $i$ ranges from 1 to $\mathrm{n}$. The background attention mask pays attention to the part that is unchanged before and after image generation, and this part is multiplied with the input CBCT images to obtain an output image. The foreground attention mask pays attention to the image part that changes before and after generation. A total of $n-1$ output images are obtained by element-wise multiplication of $\mathrm{n}-1$ foreground attention masks and $\mathrm{n}-1$ content masks. These $\mathrm{n}$ output images are added, thus obtaining the final sCT images.

The $G_{C B C T-C T}$ of AGGAN generates $\mathrm{sCT}$ images through Eq. (1).

$$
S_{C T}=\sum_{f=1}^{n-1}\left(C_{C T} * A_{C T}^{f}\right)+I_{C B C T} * A_{C T}^{b}
$$

where $C_{C T}$ is the content mask, $A_{C T}^{f}$ is the foreground attention mask, $A_{C T}^{b}$ is the background attention mask, $I_{C B C T}$ is the input CBCT images, and $S_{C T}$ is the generated sCT images. In this study, n was set to 10 .

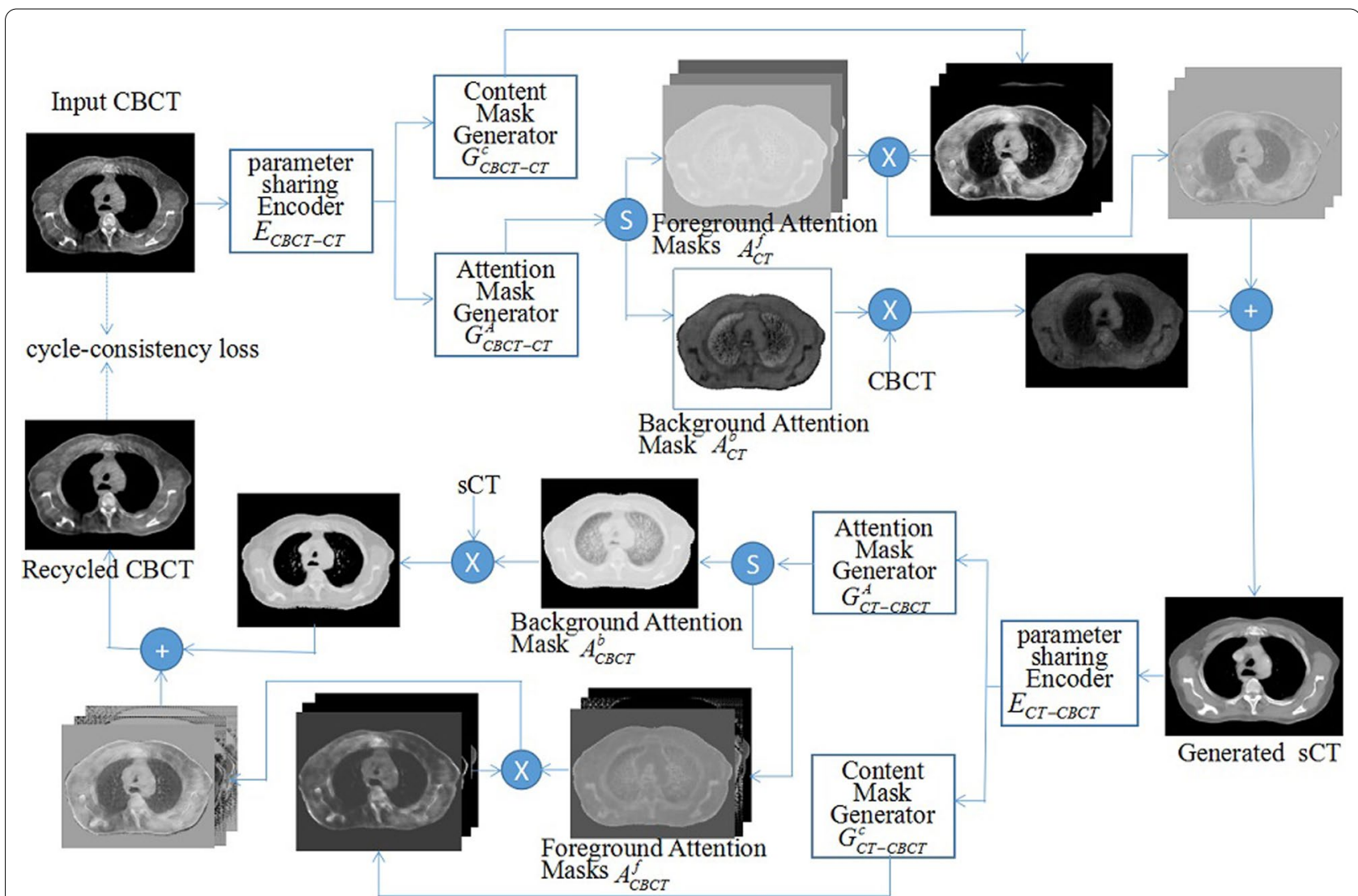

Fig. 1 Framework of the proposed AGGAN, which contains two attention-guided generators $G_{C B C T-C T}$ and $G_{C T-C B C T}$. We show one cycle in this figure, i.e., $\mathrm{CBCT} \rightarrow \mathrm{sCT} \rightarrow \mathrm{rCBCT} \approx \mathrm{CBCT}$. Each generator such as $\mathrm{G}_{C B C T-C T}$ consists of a parameter sharing encoder $E_{C B C T-C T}$, a content mask generator $G_{C B C T-C T}^{C}$ and an attention mask generator $G_{C B C T-C T}^{A}$. The proposed model is constrained by the cycle-consistency loss. The symbols $\oplus, \otimes$ and $\odot$ denote element-wise addition, element-wise multiplication and channel-wise Softmax respectively 
The generator $G_{C T-C B C T}$ generates rCBCT images through Eq. (2).

$$
R_{C B C T}=\sum_{f=1}^{n-1}\left(C_{C B C T} * A_{C B C T}^{f}\right)+S_{C T} * A_{C B C T}^{b}
$$

Corresponding to the variables in Eq. (1), $C_{C B C T}$, $A_{C B C T}^{f}$, and $A_{C B C T}^{b}$ in Eq. (2) denote the content, foreground attention, and background attention masks in $G_{C T-C B C T}$, respectively. $S_{C T}$ is the sCT images gained from Eq. (1), and $R_{C B C T}$ is the generated rCBCT images.

The adversarial loss of the neural network uses the LSGAN (Least Squares GAN) model [46] as shown in Eq. (3) and (4). $D_{C T}$ distinguishes sCT from CT and aims to classify sCT with label 0 from $\mathrm{CT}$ with label 1 . Differently, $G_{C B C T-C T}$ attempts to make sCT as close as possible to $\mathrm{CT}$ and aims to output 1 for $\mathrm{sCT}$ after the discriminator. The loss functions of the discriminators and generators are the minimum and $L_{G A N-G_{C B C T-C T}}$, respectively.

$$
\begin{aligned}
L_{G A N-D_{C T}}= & \frac{1}{2 m} \sum_{i=1}^{m}\left[\left(D_{C T}\left(I_{C T}^{i}\right)-1\right)^{2}\right. \\
& \left.+D_{C T}\left(G_{C B C T-C T}\left(I_{C B C T}^{i}\right)\right)^{2}\right] \\
L_{G A N-G_{C B C T-C T}} & =\frac{1}{2 m} \sum_{i=1}^{m}\left(D_{C T}\left(G_{C B C T-C T}\left(I_{C B C T}^{i}\right)-1\right)\right)^{2}
\end{aligned}
$$

where $m$ is the number of trained images and and $I_{C B C T}^{i}$ are the $i$ th $C T$ and $i$ th $C B C T$ images, respectively. The loss functions of $D_{C B C T}$ and $G_{C T-C B C T}$ are similar to those in Eqs. (3) and (4).

$$
\begin{aligned}
L_{G A N-D_{C B C T}}= & \frac{1}{2 m} \sum_{i=1}^{m}\left[\left(D_{C B C T}\left(I_{C B C T}^{i}\right)-1\right)^{2}\right. \\
& \left.+D_{C B C T}\left(G_{C T-C B C T}\left(I_{C T}^{i}\right)\right)^{2}\right] \\
L_{G A N-G_{C T-C B C T}}= & \frac{1}{2 m} \sum_{i=1}^{m}\left(D_{C B C T}\left(G_{C T-C B C T}\left(I_{C T}^{i}\right)-1\right)\right)^{2}
\end{aligned}
$$

The generative adversarial loss is

$$
\begin{aligned}
L_{G A N}= & L_{G A N-D_{C T}}+L_{G A N-G_{C B C T-C T}} \\
& +L_{G A N-D_{C B C T}}+L_{G A N-G_{C T-C B C T}}
\end{aligned}
$$

The neural network still can map images from one domain to several domains on the basis of generative adversarial loss only. These domains share the same distribution characteristics, which cannot ensure that the learned generator can map the input $\mathrm{CBCT}$ images to the desired CT images. A cycle-consistency loss needs to be added to decrease the mapping function spaces as much as possible; this loss requires the minimum difference between the input $\mathrm{CBCT}$ and $\mathrm{rCBCT}$ images and the minimum difference between the input $\mathrm{CT}$ and $\mathrm{rCT}$ images.

$$
\begin{aligned}
& L_{c y c l e-C B C T}=\frac{1}{m} \sum_{i=1}^{m}\left|G_{C T-C B C T}\left(G_{C B C T-C T}\left(I_{C B C T}^{i}\right)\right)-I_{C B C T}^{i}\right| \\
& L_{c y c l e-C T}=\frac{1}{m} \sum_{i=1}^{m}\left|G_{C B C T-C T}\left(G_{C T-C B C T}\left(I_{C T}^{i}\right)\right)-I_{C T}^{i}\right| \\
& L_{c y c l e}=L_{c y c l e-C B C T}+L_{c y c l e-C T}
\end{aligned}
$$

$G_{C B C T-C T}$ generates CT images from the input CBCT images. If $C T$ images are inputted into $G_{C B C T-C T}$, then the difference between the generated $\mathrm{CT}$ image and the input $\mathrm{CT}$ images should be small as possible, and it will be constrained by identity loss.

$$
\begin{aligned}
& L_{i d t-C T}=\frac{1}{m} \sum_{i=1}^{m}\left|G_{C B C T-C T}\left(I_{C T}^{i}\right)-I_{C T}^{i}\right| \\
& L_{i d t-C B C T}=\frac{1}{m} \sum_{i=1}^{m}\left|G_{C T-C B C T}\left(I_{C B C T}^{i}\right)-I_{C B C T}^{i}\right| \\
& L_{i d t}=L_{i d t-C T}+L_{i d t-C B C T}
\end{aligned}
$$

The total loss function is the sum of the three loss functions.

$$
L=L_{G A N}+\lambda_{c y c l e} * L_{c y c l e}+\lambda_{i d t} * L_{i d t}
$$

In the experiment, $\lambda_{c y c l e}$ was set 10 , and $\lambda_{i d t}$ was set 5 .

\section{Neural network training}

In conventional thoracic $\mathrm{CT}$ images, a few pixels have $\mathrm{HU}>1500$. In this study, the $\mathrm{HU}$ value of images was clipped to $[-1000,1500] \mathrm{HU}$, and those exceeding $1500 \mathrm{HU}$ were set to $1500 \mathrm{HU}$. Afterward, the pixel values were scaled to $[-1,1]$ and inputted into the neural network. In consideration of the requirements on GPU memory and training efficiency of the neural network, 2D axial slices of the $\mathrm{CT}$ images were used and resized to $256 \times 256$ for training. Kida [26] pointed out that $2 \mathrm{D}$ axial slices of $\mathrm{CT}$ images can generate good sCT images and do not cause structural discontinuity in coronal and sagittal views. The training dataset contained thoracic $\mathrm{CT}$ and $\mathrm{CBCT}$ images of 136 patients and involved 12,784 slices of CBCT and CT images. The testing dataset contained 3,196 slices 
of CBCT images of 34 patients. During the training of AGGAN and cycleGAN, the CBCT and CT images were shuffled in each epoch so that they could be trained through unpaired data. The pix 2 pix network was trained using the paired $\mathrm{CBCT}$ and $\mathrm{CT}$ images.

In AGGAN, the downsampling of the generator consisted of (a) one convolution layer with a $7 \times 7$ kernel with a stride of 1 and 64 channels, (b) two convolution layers with a $3 \times 3$ kernel with a stride of 2 and 128,256 channels, and (c) 9 residual blocks with a $3 \times 3$ kernel with a stride of 1 and 64 channels. The upsampling involved two independent branches of content and attention masks. The first of the two branches had two deconvolution layers with a $3 \times 3$ kernel with a stride of 2 and 128, 64 channels using the ConvTranspose $2 \mathrm{~d}$ function. The last layer of the content mask was a $7 \times 7$ convolution layer with a stride of 1 and 9 channels. The last layer of the attention mask was a $1 \times 1$ convolution layer with a stride of 1 and 10 channels. Instance normalization was performed after each convolution layer except for the last layer, and ReLU was used as the activation function [47]. The discriminator used PatchGAN in pix2pix [41], the mean of all patches in an image was calculated to judge whether the entire image was true or false. The batch size was set to 1 during training, and the Adam optimizer was used for optimization. The momentum was set to $\beta 1=0.5$ and $\beta 2=0.999$, and a total of 100 epochs were established. The initial learning rate of Adam was set to 0.0001, and after 50 epochs the learning rate started linearly decaying to 0 . Pix2pix [41] and cycleGAN [42] were trained in the way indicated in the original paper, and the number of epochs was set to 100 . The neural networks were implemented in the PyTorch framework with Pycharm software and the training was done on a NVIDIA 2080 Ti Graphical Processing Unit(GPU). The training computation time for the pix2pix, cycleGAN and AGGAN was $428 \mathrm{~h}, 655 \mathrm{~h}, 732 \mathrm{~h}$ respectively. Once trained, the network is able to generate $\mathrm{sCT}$ from CBCT images with mean speed of 141 slices/ min, 142 slices/min and 133 slices/min for the pix2pix, cycleGAN and AGGAN respectively. That is to say the trained networks can generate $\mathrm{SCT}$ for a patient (usually less than 100 slices) within one minute.

\section{Evaluation}

A side-by-side comparison of true CT images, $\mathrm{CBCT}$ and sCT generated by pix2pix, cycleGAN, AGGAN was performed at the mediastinal window of $[-400,400]$ HU and lung window of $[-1200300] \mathrm{HU}$. The HU histogram distribution of one patient's 3D images were also compared. The $\mathrm{sCT}$ images of patients generated from neural networks in testing dataset were quantitatively evaluated by computing the MAE, SSIM, and peak signal-to-noise ratio (PSNR) with deformed CT images as the reference. An intensity-modulated radiation therapy phantom (002LFC, CIRS, USA) was scanned by CT and $\mathrm{CBCT}$. The CBCT and CT images of the phantom were aligned through $3 \mathrm{D}$ rigid registration. The image quality of the $\mathrm{SCT}$ images for phantom was quantitatively evaluated using the $\mathrm{CT}$ images as reference. In addition, three regions of interest (ROI) were identified on each image (lung, bone and soft tissues). The mean $\mathrm{HU}$ values with standard deviation (SD) of ROIs for test patients or phantom were calculated and compared. The image quality indices were compared by paired Wilcoxon rank test and the statistical significance level was set at $P<0.05$.

To verify the dose calculation accuracy, the treatment plans of the 34 test patients were copied to deformed CT images, $\mathrm{CBCT}$ and $\mathrm{sCT}$ images in the treatment planning system (Monaco5.1, Elekta). The dose distribution were calculated directly without optimized in these images. Using the dose distribution of deformed CT images as reference, the $3 \mathrm{D}$ gamma passing rates of the dose distributions on $\mathrm{CBCT}$ and $\mathrm{sCT}$ images were calculated under different criteria (distance to agreement and relative dose difference). In addition, a treatment plan was designed based on the phantom to simulate lung cancer radiotherapy. Volumetric-modulated arc therapy (VMAT) was adopted. The radiation field was rotated by $360^{\circ}$, and target 5000 cGy of the prescription dose was applied. Subsequently, dose distribution was calculated and compared in $\mathrm{CT}, \mathrm{CBCT}$ and $\mathrm{SCT}$ images of the phantom.

\section{Results \\ Comparison of image quality and preservation of the anatomical structure}

The sCT images generated by different neural networks from the CBCT of the same patient in the test are shown in Fig. 2. Each row shows images of the same slice. From top to bottom, the rows denote axial slices of the mediastinal window display, axial slices of the lung window display, and coronal and sagittal images. The first and second rows show the same slice. The columns from left to right display $\mathrm{CBCT}, \mathrm{CT}$, and $\mathrm{SCT}$ images generated by pix2pix, cycleGAN, and AGGAN, respectively. Serious streaking and shading artifacts were observed at the chest wall and other sites of the original CBCT images, respectively, due to the influence of the respiratory movement of patients during scanning. The lung window shows that the lung was relatively dark, and the HU value in CBCT had serious distortion. Most of the artifacts in the $\mathrm{sCT}$ images generated by pix2pix were eliminated, but several anatomical structures, especially the bone, cavity, and lung marking regions (red arrows), in the 


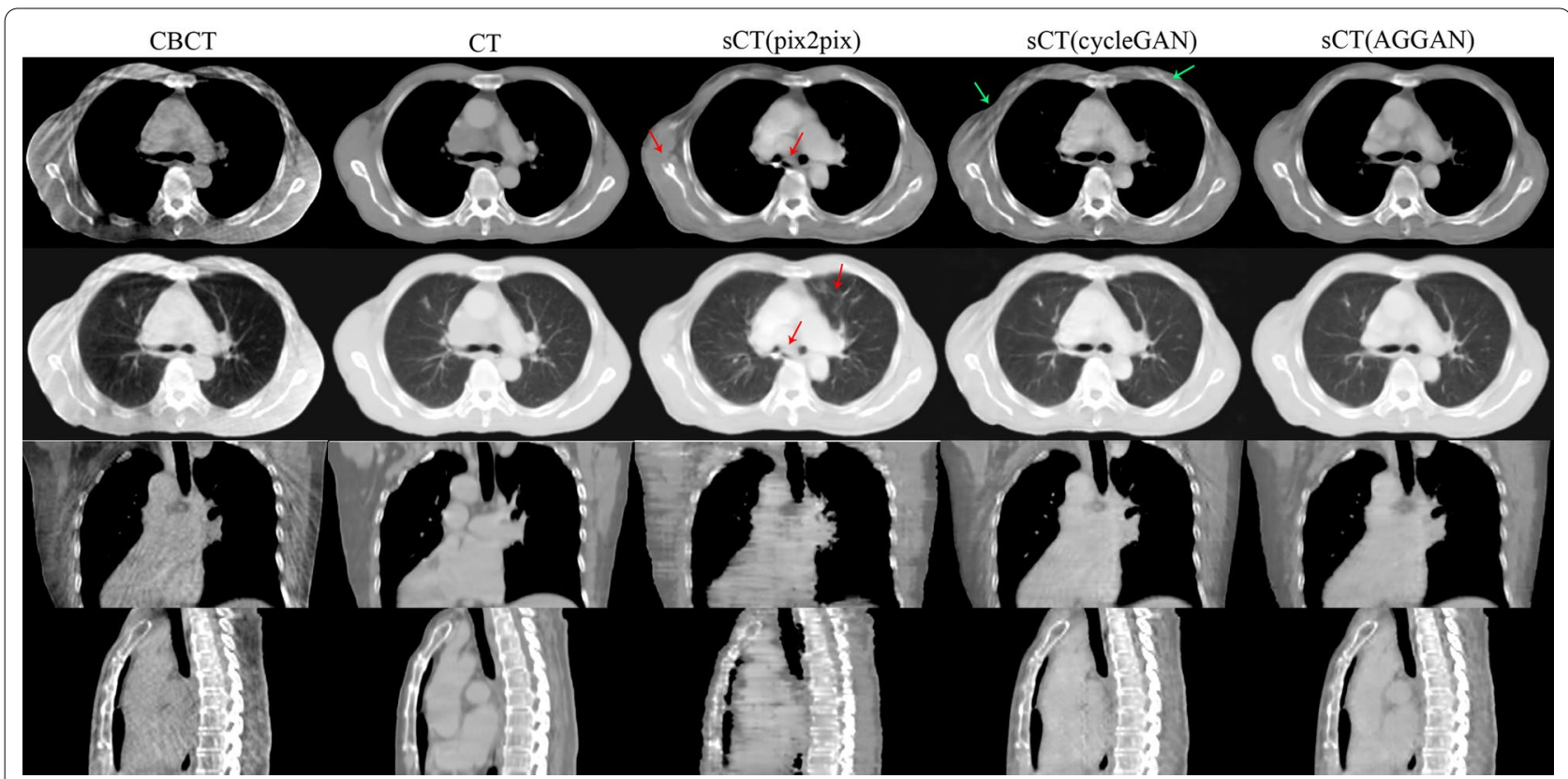

Fig. 2 Quality comparison of CBCT, CT, and sCT images generated by three neural networks for the same patient in axial, coronal, and sagittal images. The display window in second row is [-1200 300] HU (lung window), and display window in other rows is [-400 400] HU

images were destroyed. The coronal and sagittal images presented serious image distortions. The sCT images generated by cycleGAN maintained several streaking artifacts on the axial slices. In particular, the chest wall (green arrow in the images) had serious artifacts, but the anatomical structure was preserved well. The coronal and sagittal images revealed good tissue continuity. Most artifacts in the $\mathrm{SCT}$ images generated by AGGAN were eliminated, and the anatomical structures were well-preserved. The quality of the coronal and sagittal images was also improved.

The image histograms of the 3D images for patients shown in Fig. 2 were analyzed (Fig. 3). In Fig. 3, the $x$-axis denotes the $\mathrm{HU}$ value, and the $\mathrm{y}$-axis denotes the number of occurrences of $\mathrm{HU}$ values in the 3D CT images. The $\mathrm{HU}$ value distributions of the $\mathrm{CBCT}$ and $\mathrm{CT}$ images differed considerably. The $\mathrm{HU}$ value of the $\mathrm{SCT}$ images generated by neural networks showed a similar distribution as that of the real CT images. The distribution curves of the $\mathrm{CT}$ and $\mathrm{SCT}$ images had an evident peak at about $-800 \mathrm{HU}$, which is the $\mathrm{HU}$ value distribution of the lung. However, such a peak was absent in the CBCT images. The HU value distribution of the $\mathrm{SCT}$ images generated by AGGAN was the closest to that of the CT images.

The $\mathrm{SCT}$ images generated from the CBCT images of test patients in axial view are shown in Fig. 4. The first four rows from the top to the bottom are axial slice images of different patients, and the fifth row is the lung window display of the fourth row. The same row shows the same slice of images of patients. The generated sCT images in coronal and sagittal views are shown in Fig. 5. Similar to the results in Fig. 2, the CBCT images included many artifacts, and the HU value had serious distortion. Pix2pix eliminated many artifacts but destroyed the anatomical structures, which mainly included bone tissues, cavities, lung marking, liver, and heart edges (red arrows). The sCT images generated by cycleGAN had well-preserved anatomical structures but retained several streaking artifacts from the CBCT images (green arrow). The $\mathrm{SCT}$ images generated by AGGAN reduced more artifacts than cycleGAN, and preserved the anatomical structures well.

The quantitative analysis results of $\mathrm{CBCT}$ and $\mathrm{sCT}$ on image quality for testing patients are listed in Table 2 . The image quality indices of all sCT images are significantly improved compared with original $\mathrm{CBCT}$ images $(P<0.05)$. The AGGAN achieves the best image quality with the smallest MAE, largest SSIM and PSNR. The image quality indices of $\mathrm{sCT}$ generated from cycleGAN and AGGAN are both better than pix2pix. Compared with cycleGAN, sCT images generated from AGGAN show significant superiority in MAE and PSNR $(P<0.05)$. There are no significant difference in SSIM between sCT images generated from cycleGAN and AGGAN $(P=0.261)$. The sCT images of patients generated by AGGAN show the best image quality. The mean $\mathrm{HU}$ values of ROIs on CT, CBCT and sCT images for patients 


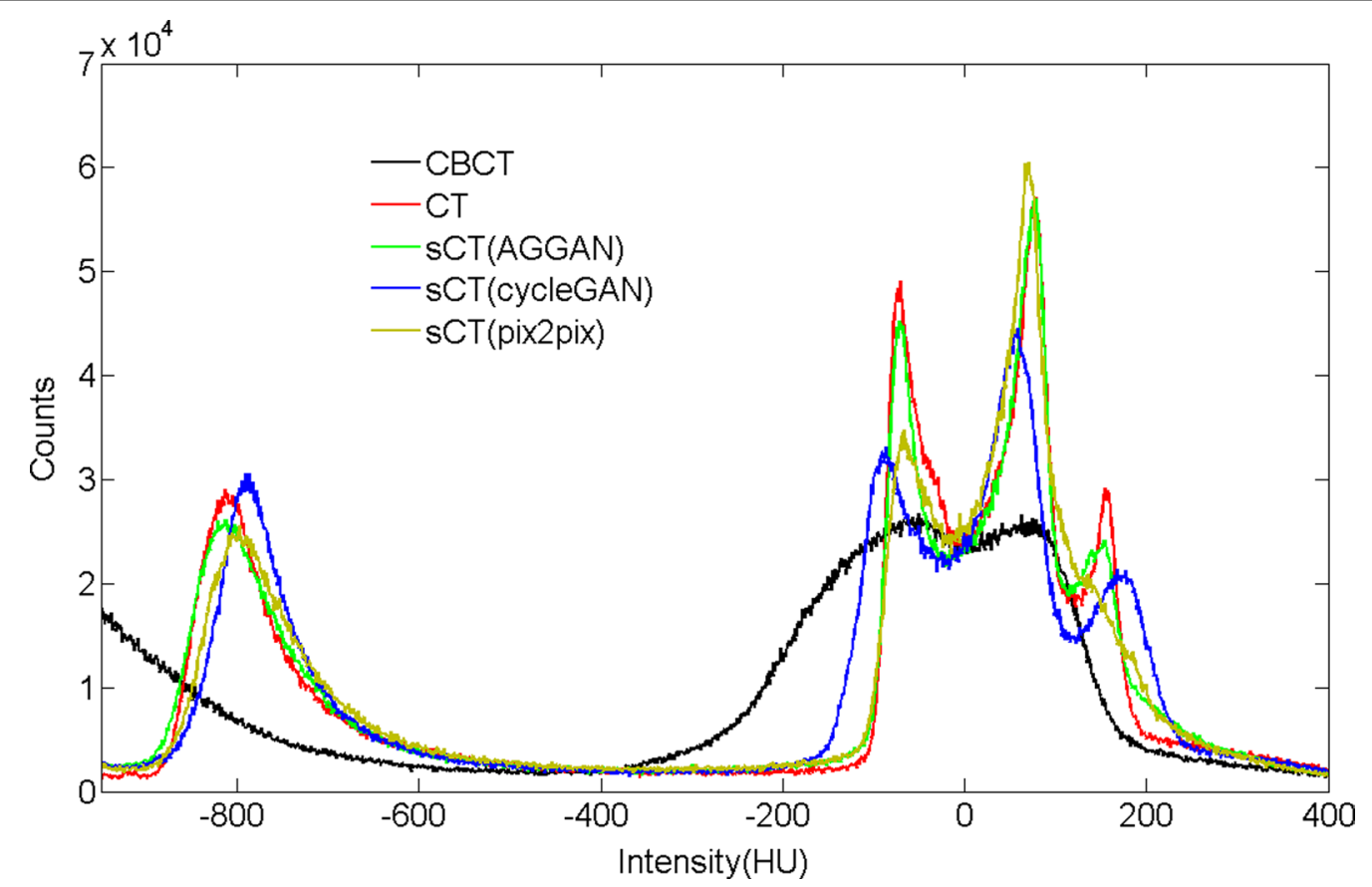

Fig. 3 Histogram distribution curves of the $\mathrm{HU}$ values of $3 \mathrm{D} C \mathrm{CT}, \mathrm{CBCT}$, and $\mathrm{SCT}$ images generated by three neural networks

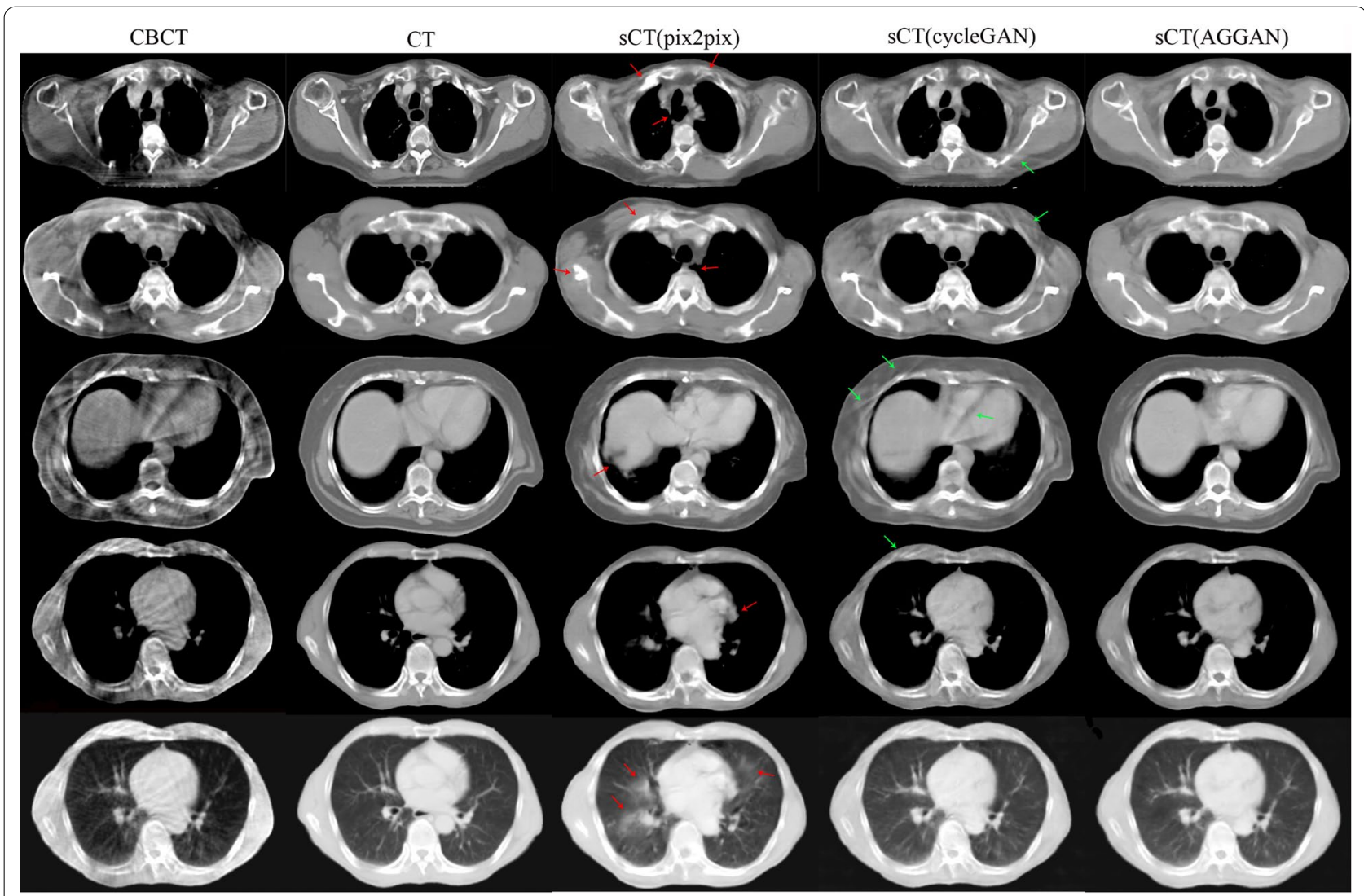

Fig. 4 Comparison of sCT images generated by the three neural networks from CBCT images of patients in the test. The display window in bottom row is [ -1200300$] \mathrm{HU}$ (lung window), and display window in other rows is [- 400400$] \mathrm{HU}$ 


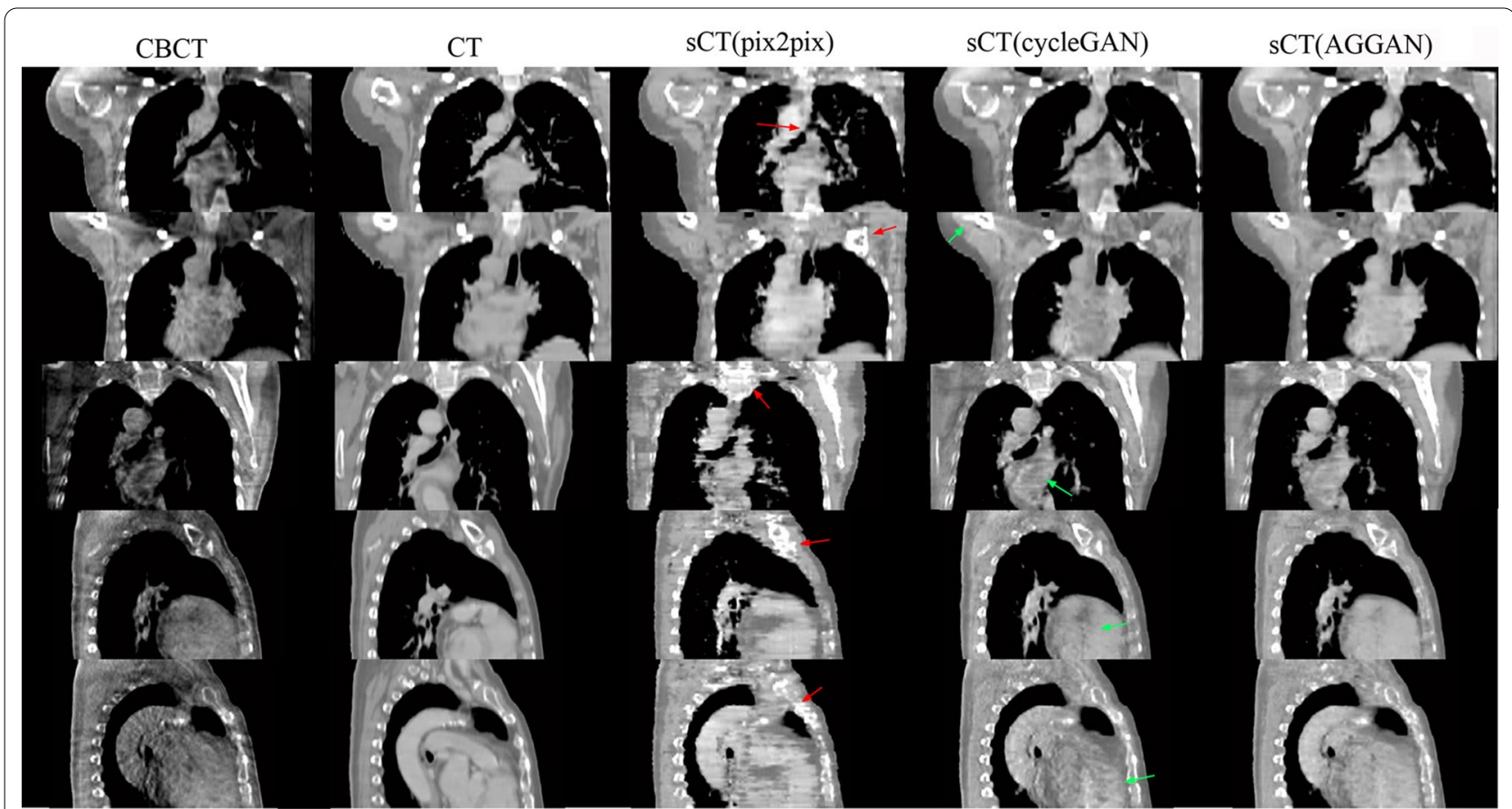

Fig. 5 Comparison of sCT images generated by the three neural networks from test CBCT images in coronal (the top three rows) and sagittal (the bottom two rows) views. The display window is [-400 400] HU

Table 2 Image quality indices of CBCT and SCT images generated by the three neural networks

\begin{tabular}{lllll}
\hline & CBCT & sCT (pix2pix) & sCT (cycleGAN) & sCT (AGGAN) \\
\hline MAE (HU) & $92.8 \pm 16.7$ & $53.4 \pm 9.34$ & $47.1 \pm 6.45$ & $43.5 \pm 6.69$ \\
SSIM (\%) & $78.3 \pm 6.34$ & $88.1 \pm 7.12$ & $93.2 \pm 4.17$ & $93.7 \pm 3.88$ \\
PSNR (dB) & $21.6 \pm 2.81$ & $26.8 \pm 2.73$ & $28.3 \pm 2.04$ & $29.5 \pm 2.36$ \\
\hline
\end{tabular}

are listed in Table 3. The mean HU values of lung, bone and soft tissue on CBCT images are significantly less than that on $\mathrm{CT}$ images $(P<0.05)$. In addition, the mean $\mathrm{HU}$ values of ROIs on CBCT images fluctuated greatly, leading to a large SD number. The mean $\mathrm{HU}$ values of ROIs on $\mathrm{SCT}$ images generated from three networks are close to that on $\mathrm{CT}$ images. There are no significant difference on mean $\mathrm{HU}$ values of ROIs among CT, sCT generated from pix2pix, cycleGAN and AGGAN.

\section{Dose calculation}

The relative dose distribution calculated in treatment plans on $\mathrm{CT}, \mathrm{CBCT}$ and $\mathrm{SCT}$ images for patients are shown in Fig. 6. Using the dose distribution calculated in the CT images as a reference, the absolute gamma analysis distribution of each corresponding image under the criteria $2 \mathrm{~mm} / 2 \%$ are shown in Fig. 7. The dose distributions in the original CBCT images remained highly divergent compared with the reference. There are large regions where the gamma index is greater than 1 in $\mathrm{CBCT}$ images. The dose distribution of the $\mathrm{sCT}$ images are close to the reference, and the areas with a gamma index greater than 1 are greatly reduced. The statistical analysis of $3 \mathrm{D}$ gamma passing rates with different standards for the 34 testing

Table 3 The mean HU values of ROIs on CT, CBCT and SCT images for patients

\begin{tabular}{lrrrrr}
\hline & \multicolumn{1}{l}{ Mean HU value of ROIs (mean \pm SD) } & & & \\
\cline { 2 - 6 } & CT & CBCT & sCT (pix2pix) & sCT (cycleGAN) & sCT (AGGAN) \\
\hline Lung $(H U)$ & $-722.6 \pm 46.1$ & $-853.9 \pm 85.2$ & $-708.2 \pm 49.4$ & $-704.8 \pm 40.8$ & $-710.3 \pm 42.7$ \\
Bone $(H U)$ & $217.4 \pm 23.8$ & $67.1 \pm 42.7$ & $223.0 \pm 27.5$ & $208.7 \pm 25.1$ & $211.7 \pm 27.2$ \\
Soft tissue $(H U)$ & $13.7 \pm 16.2$ & $-116.7 \pm 75.0$ & $14.2 \pm 15.1$ & $9.6 \pm 14.7$ & $12.4 \pm 15.7$ \\
\hline
\end{tabular}




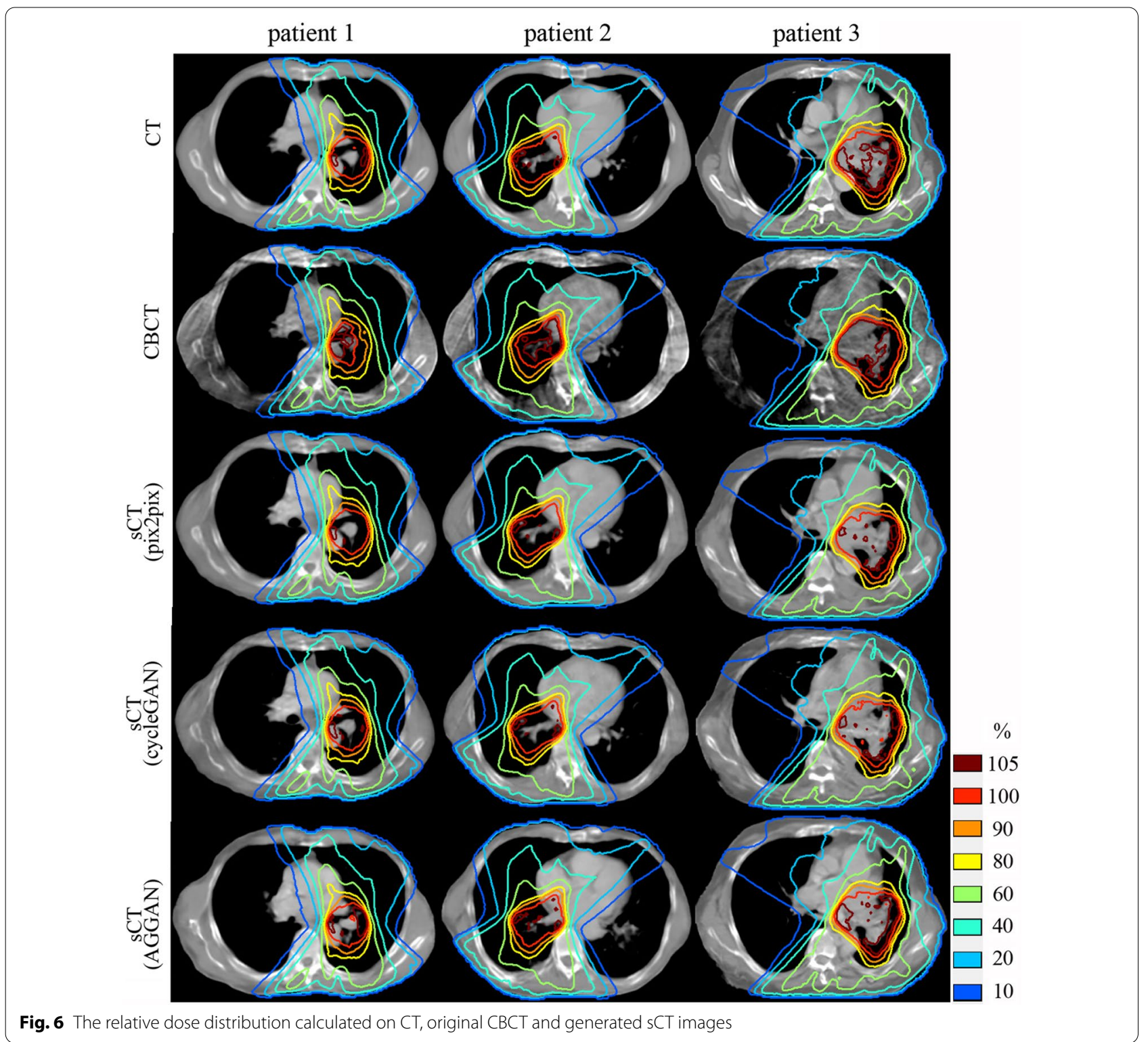

patients are shown in Table 4. The gamma passing rates of $\mathrm{sCT}$ images generated from three methods were significantly improved under all criteria compared with that of original CBCT $(P<0.05)$. Under the criteria $1 \mathrm{~mm} / 1 \%$ and $2 \mathrm{~mm} / 2 \%$, the gamma passing rates of cycleGAN and AGGAN were significantly increased compared with that of pix2pix $(P<0.05)$, but no significant differences were observed under $3 \mathrm{~mm} / 3 \%$ criteria $(P=0.165)$. There are no significant differences for the gamma passing rates between cycleGAN and AGGAN under criteria $2 \mathrm{~mm} / 2 \%$ or $3 \mathrm{~mm} / 3 \%(P=0.214$ and $P=0.345)$. However, AGGAN got significantly higher passing rates than cycleGAN under the $1 \mathrm{~mm} / 1 \%$ criteria $(P<0.05)$. In conclusion, the SCT images generated by AGGAN obtained the optimal dose calculation accuracy in radiotherapy for testing patients.

\section{A phantom study}

The CT, CBCT, and sCT images of the phantom are shown in Fig. 8. The images from the left to the right are the soft tissue window display, lung window display, and the image difference. The image difference was obtained by subtracting the CT image from each image. The dark region represents the HU value of the image part that is lower than that of the CT images, and the bright region represents the $\mathrm{HU}$ value of the 


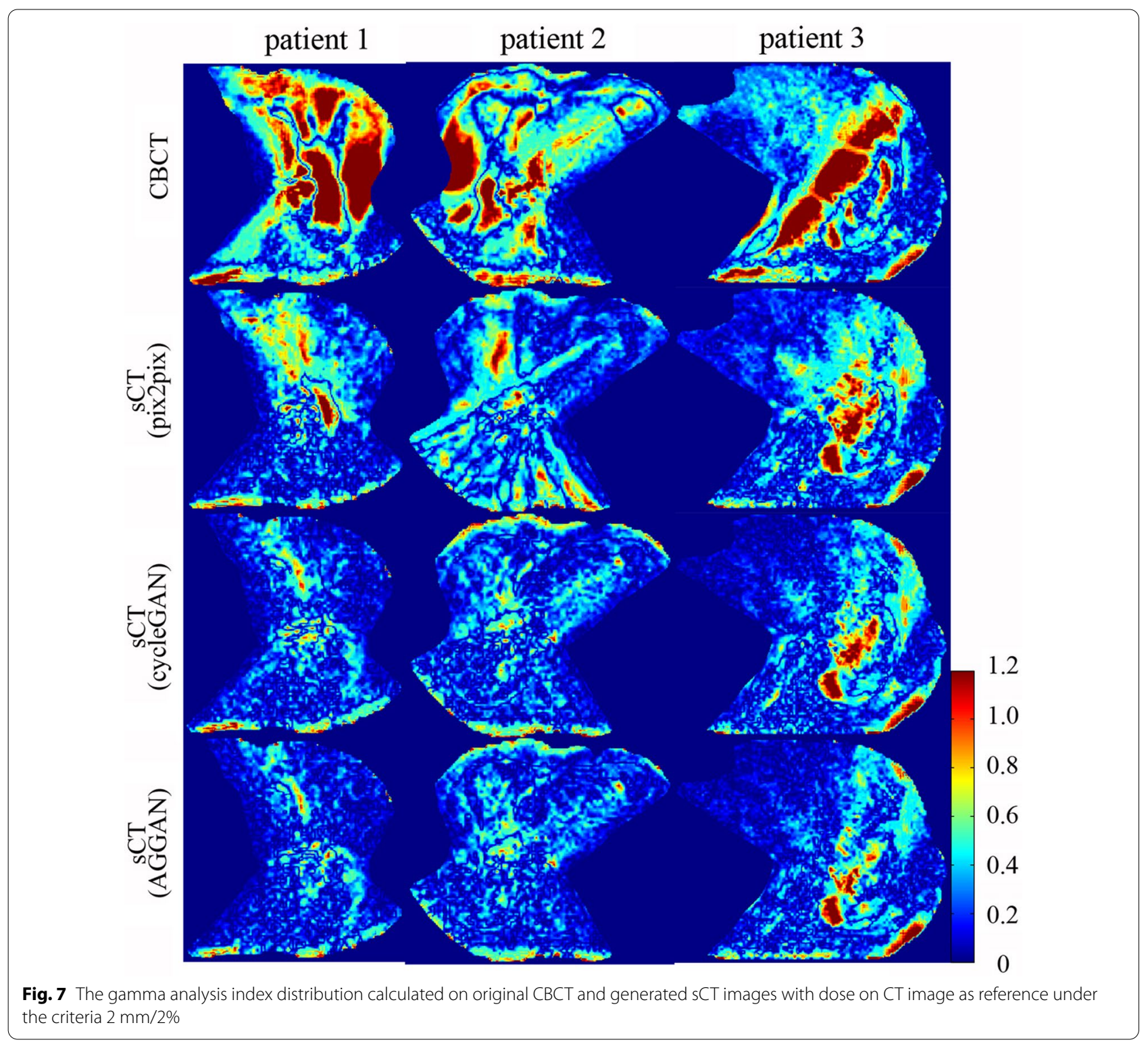

Table 4 The 3D gamma passing rates of dose distribution in $\mathrm{CBCT}$ and $\mathrm{SCT}$ images for patients

\begin{tabular}{|c|c|c|c|}
\hline & \multicolumn{3}{|c|}{ Gamma criteria } \\
\hline & $1 \mathrm{~mm} / 1 \%$ & $2 \mathrm{~mm} / 2 \%$ & $3 \mathrm{~mm} / 3 \%$ \\
\hline СBCT (\%) & $50.1 \pm 9.04$ & $84.4 \pm 5.81$ & $92.8 \pm 3.86$ \\
\hline sCT-pix2pix (\%) & $75.3 \pm 4.50$ & $96.7 \pm 2.26$ & $99.4 \pm 0.67$ \\
\hline sCT-cycleGAN (\%) & $89.3 \pm 3.81$ & $98.2 \pm 2.09$ & $99.7 \pm 0.44$ \\
\hline sCT-AGGAN (\%) & $91.4 \pm 3.26$ & $98.6 \pm 1.78$ & $99.7 \pm 0.39$ \\
\hline
\end{tabular}

image that is higher than that of the CT images. The original CBCT image of the phantom had large differences with the CT images. The lung tissues were relatively dark, and the soft tissue regions showed irregular shading. The $\mathrm{sCT}$ image generated by pix 2 pix destroyed the original structures seriously. However, the sCT images generated by cycleGAN and AGGAN retained the structures of the phantom well. The $\mathrm{sCT}$ images generated by cycleGAN showed a dark region at the right side of the lung, and the $\mathrm{HU}$ value of the cylinder inserted into the lung was larger than that in $\mathrm{CT}$. The sCT images generated by AGGAN showed no large differences. The HU profile on one straight (red straight) of images is shown in Fig. 9, where considerable differences can be observed between the CBCT and $\mathrm{CT}$ images. The $\mathrm{HU}$ value of the CBCT images in the lung was close to zero. The HU value distribution 


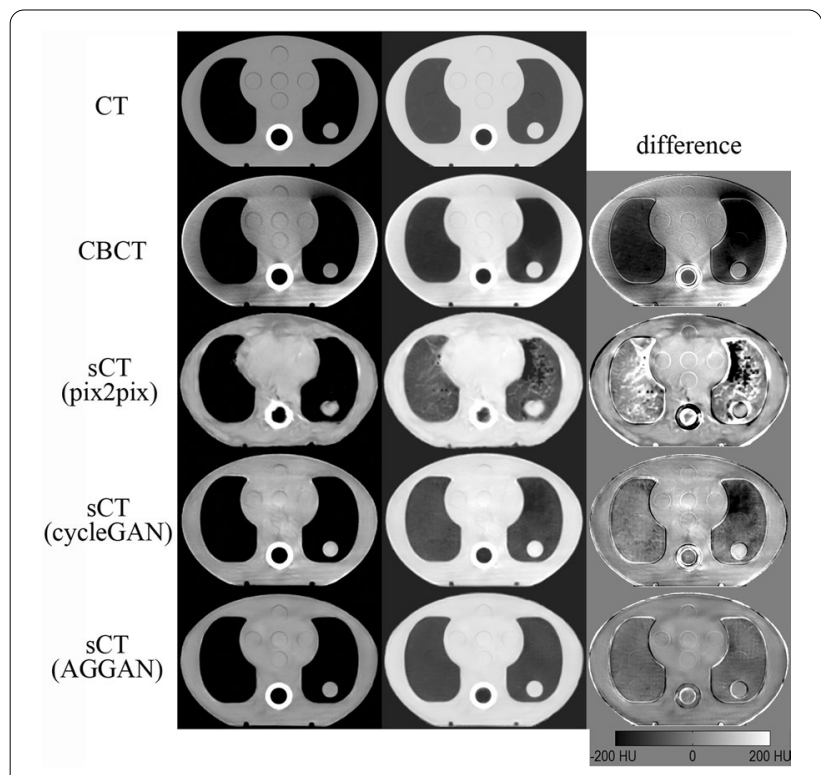

Fig. 8 Quality comparison of $C T, C B C T$, and $\mathrm{SCT}$ images generated by the three neural networks. The display window in left column is [ -400400$] \mathrm{HU}$, and in middle column is [- 1200 300] HU

of the sCT images generated by AGGAN was the closest to that of the CT images.

The CT images of the phantom were used as the reference, and the MAE, SSIM, and PSNR of the different images were calculated. The results are listed in Table 5 . The MAE of the $\mathrm{SCT}$ generated by AGGAN was the lowest $(23.2 \mathrm{HU})$, but its SSIM and PSNR were the highest (0.944 and 30.2, respectively). The SSIM (0.938) of the $\mathrm{sCT}$ images generated by cycleGAN was close to that of AGGAN, but the MAE (32.6 HU) was higher. Pix2pix exerted a poor experimental effect on the phantom, as manifested by the lower SSIM and PSNR than those of the original CBCT images. The MAE of pix2pix was hardly improved compared with that of the CBCT images. In addition, the lung, bone and soft tissue were contoured and the mean HU value of these ROIs were calculated, as shown in Table 6. The mean HU value of sCT generated by AGGAN are closest to that of CT on three ROIs. In the phantom experiment, the $\mathrm{SCT}$ images generated by AGGAN showed the best quality.

The calculated dose distribution in the phantom is shown in Fig. 10. The upper left part presents diagrams of the irradiation field and the target area (green profile), and the upper right part presents the calculated dose distribution in the CT images. The lower left and lower right parts show the relative dose difference distributions calculated on $\mathrm{CBCT}$ and $\mathrm{sCT}$ images generated from pix2pix, cycleGAN, AGGAN respectively. The relative dose difference distribution was obtained through the calculated dose from CBCT or sCT images minus the dose in the $\mathrm{CT}$ images and divided by the maximum dose in the CT images. Dark regions indicate that the calculated dose is lower than the reference dose, and the bright regions indicate that the calculated dose is higher than the reference dose. The high-dose regions (close to the target) in the CBCT images had large differences compared with

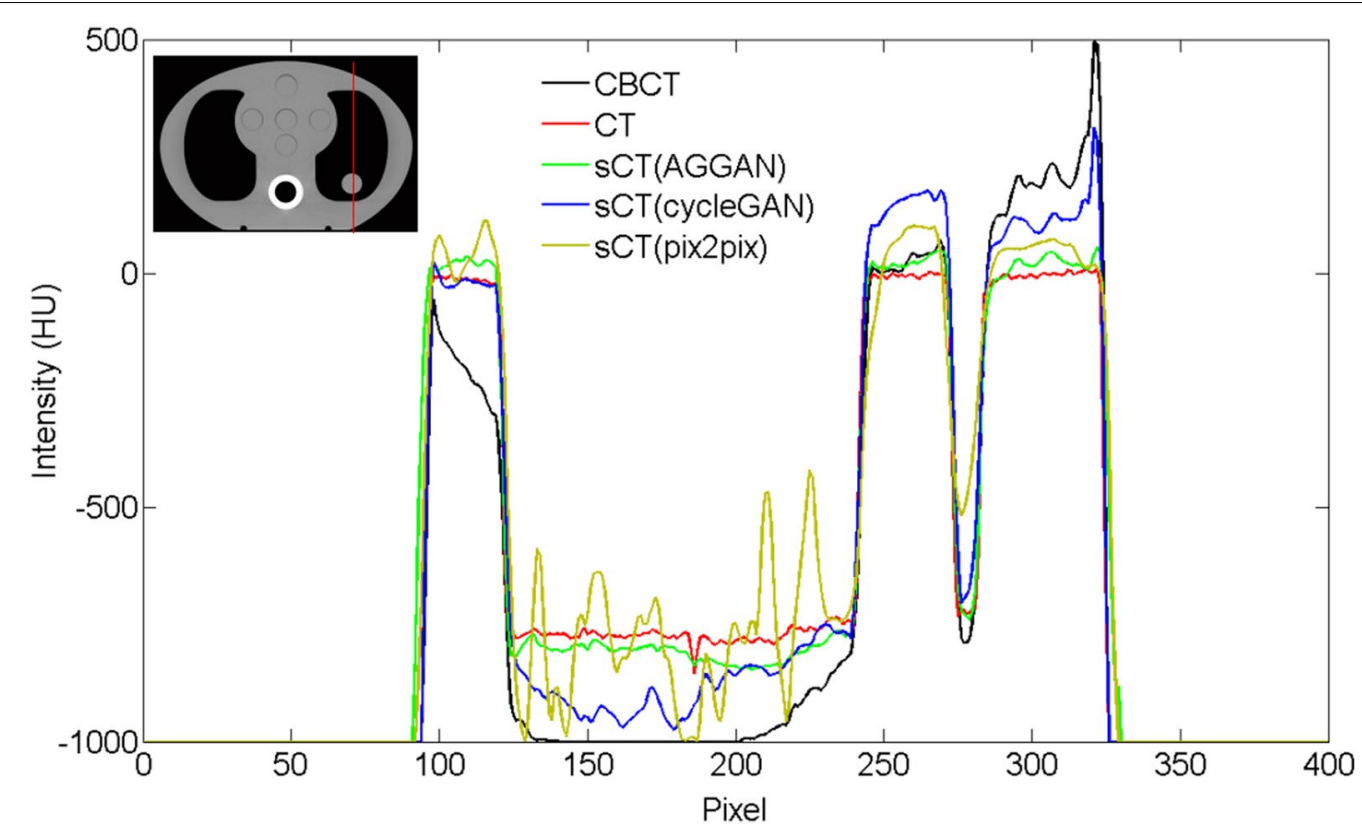

Fig. $9 \mathrm{HU}$ value distribution of images on the red straight 
Table 5 Image quality indices of CBCT and SCT images in the phantom

\begin{tabular}{lclll}
\hline & CBCT & sCT (pix2pix) & sCT (cycleGAN) & sCT (AGGAN) \\
\hline MAE(HU) & 62.9 & 62.3 & 32.6 & 23.2 \\
SSIM & 0.842 & 0.828 & 0.938 & 0.944 \\
PSNR & 25.8 & 24.0 & 28.5 & 30.2 \\
\hline
\end{tabular}

$\mathrm{CT}$. The dose difference in the $\mathrm{SCT}$ images reduced and minimum in AGGAN. The 3D gamma passing rates of the dose distributions on $\mathrm{CBCT}$ and $\mathrm{sCT}$ images with different standards were calculated (Table 7). The passing rates of dose distributions on the $\mathrm{SCT}$ images were higher than those on the CBCT images under all standards. Given the strictest standard of $1 \mathrm{~mm} / 1 \%$, the passing rate of the $\mathrm{SCT}$ images generated from AGGAN reached as high as $96.5 \%$, but that of the CBCT images was only 79.8\%. sCT images generated by AGGAN are thus conducive to calculating radiotherapy doses accurately.

\section{Discussions}

In this study, sCT images were generated from low-dose CBCT images of thoracic patients by using pix2pix, cycleGAN, and AGGAN. The paired datasets were used in pix2pix training, whereas cycleGAN and AGGAN applied unpaired training datasets. The pix 2pix reduced most of the artifacts of the original CBCT images in axial slices, but it destroyed the anatomical structures of normal tissues, resulting in image ambiguity and structural discontinuity in sagittal and coronal images. In the phantom study, pix2pix exerted great structural damages and failed to improve the image quality. The poor test results of pix2pix may be attributed to the incomplete alignment between CBCT and CT images in the training dataset. In this study, the training dataset was obtained through 3D rigid registration of $\mathrm{CT}$ and $\mathrm{CBCT}$ images. The CT and CBCT images after registration had evident local mismatching resulting from anatomical structure changes and movement of organs during the two scanning events. In particular, tissue structures, such as the trachea, esophagus, and bones, and the organ water/air filling status did not correspond to one another. Li [21] and Chen [22] implemented paired training by the Unet structure and generated sCT images based on CBCT images of patients with head and neck cancer. Given that organs in the head and neck are stationary, a good training dataset was obtained after the registration of CBCT and CT images. Liu [25] used a paired training dataset through DIR of CBCT and CT images of patients with pancreatic cancer. The images were collected from patients who received stereotactic body radiation therapy and held their breath, and relatively small differences among images were obtained. In conventional radiotherapy, thoracic $\mathrm{CBCT}$ images have serious artifacts due to respiration movement, and accurate DIR with CT images is facing a great challenge [15]. Liang [23] conducted a phantom study of the head and neck and proved that $\mathrm{SCT}$ images generated by neural networks have a more accurate anatomical structure than $\mathrm{CT}$ images obtained from DIR. Supervised learning methods, such as pix2pix, can only generate high-quality $\mathrm{sCT}$ images under the premise of accurate alignment between $\mathrm{CBCT}$ and $\mathrm{CT}$ images. Unsupervised learning methods, such as cycleGAN, do not depend on image registration results; thus, the generated $\mathrm{sCT}$ images maintain the anatomical structures well, and sagittal and coronal images have continuous structures. This finding is similar to the result of Liang [23] for head and neck images. However, the sCT images generated by cycleGAN in our experiment retained several artifacts. Given that the thoracic CBCT images contained more artifacts than the CBCT images of the head and neck due to respiration movement, cycleGAN failed to inhibit several serious artifacts, especially at the chest wall and heart with great movements. AGGAN modified the generator of cycleGAN via the background attention mask focusing on constant areas and foreground attention masks focusing on changing areas and combined them to generate the final sCT images. The quantitative evaluation of sCT images for testing patients and a phantom demonstrated that the $\mathrm{sCT}$ images generated by AGGAN achieved the best image quality, with the highest SSIM, PSNR and the lowest MAE. The accuracy of dose calculation in radiotherapy is closely related to the accuracy of HU values in CT images. The statistical analysis on 3D gamma passing rates of dose distribution demonstrated that $\mathrm{SCT}$ image generated from all the three

Table 6 The mean $\mathrm{HU}$ values of ROIs on CT, CBCT and SCT images for phantom

\begin{tabular}{|c|c|c|c|c|c|}
\hline & \multicolumn{5}{|c|}{ Mean HU value of organs (mean \pm SD) } \\
\hline & CT & СBCT & sCT (pix2pix) & sCT (cycleGAN) & sCT (AGGAN) \\
\hline Lung(HU) & -755.0 & -899.7 & -665.2 & -776.8 & -763.5 \\
\hline Bone $(\mathrm{HU})$ & 716.2 & 964.7 & 570.9 & 802.0 & 673.4 \\
\hline Soft tissue (HU) & -27.5 & 10.3 & 44.1 & 24.3 & -6.8 \\
\hline
\end{tabular}



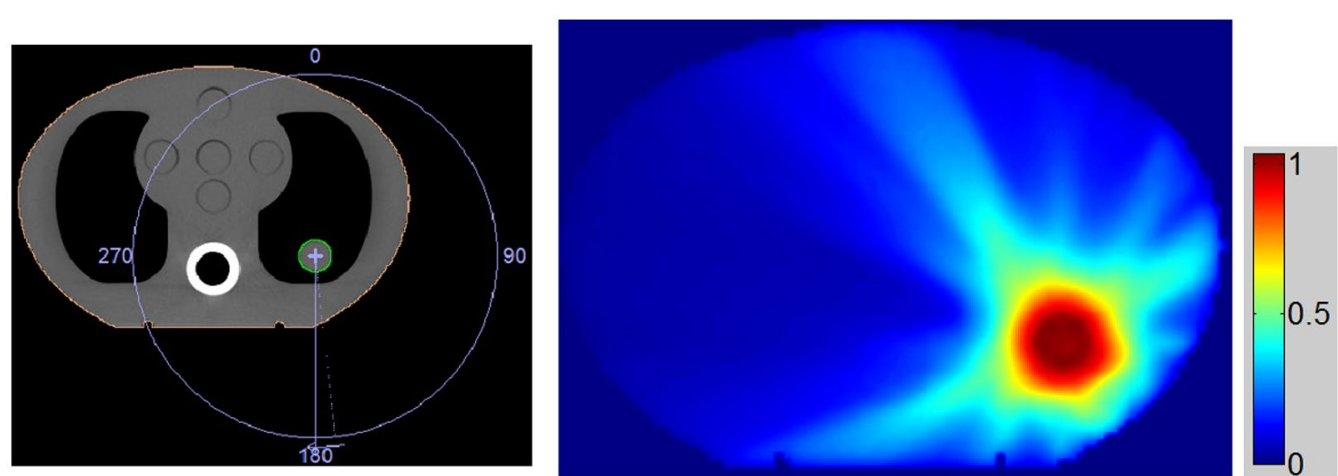

\section{Dose distribution in CT}

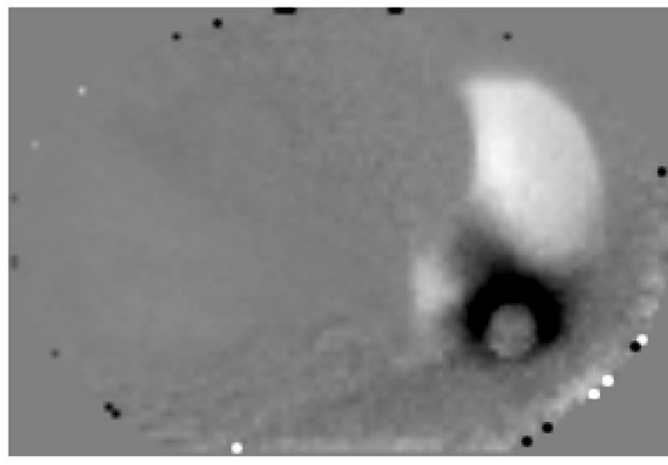

CBCT

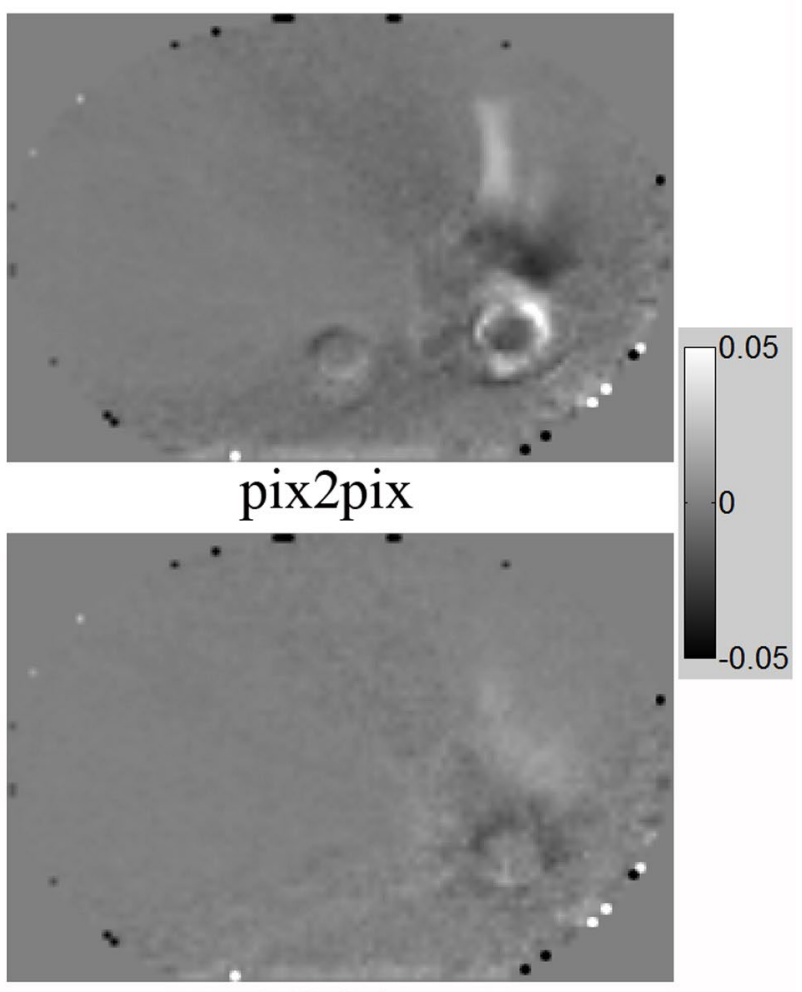

cycleGAN

\section{AGGAN}

Fig. 10 Dose distribution in CT images and distributions of relative dose differences in CBCT and SCT images genarated from pix2pix, cycleGAN and AGGAN

Table 7 3D gamma passing rates of dose distribution on CBCT and SCT images in the phantom

\begin{tabular}{llll}
\hline \multicolumn{4}{l}{ Gamma criteria } \\
\cline { 2 - 4 } & $\mathbf{1 ~ \mathbf { ~ m } / \mathbf { 1 \% }}$ & $\mathbf{2} \mathbf{~ \mathbf { m }} \mathbf{2} \%$ & $\mathbf{3} \mathbf{~ m m} \mathbf{3} \%$ \\
\hline CBCT (\%) & 79.8 & 91.6 & 96.4 \\
SCT-pix2pix (\%) & 86.4 & 97.8 & 99.2 \\
sCT-cycleGAN (\%) & 91.1 & 99.3 & 100 \\
sCT-AGGAN (\%) & 96.5 & 99.9 & 100 \\
\hline
\end{tabular}

methods significantly improved the accuracy of dose calculation compared with original CBCT image. The sCT generated by AGGAN offered the highest gamma passing rates under the strictest criteria of $1 \mathrm{~mm} / 1 \%$ compared with other methods. The sCT generated by AGGAN showed the best performance in correcting $\mathrm{HU}$ value of the image, the anatomical structures preservation and dose calculation in radiotherapy. 


\section{Conclusions}

Unpaired low-dose thoracic $\mathrm{CBCT}$ and $\mathrm{CT}$ images were trained by AGGAN. The generated high-quality $\mathrm{sCT}$ images reduced most artifacts and preserved the anatomical structures well. The sCT generated by AGGAN provided high-accuracy dose distribution calculation and can thus be applied to adaptive radiotherapy.

\section{Acknowledgements}

None.

\section{Authors' contributions}

LG and KX participated in the design of the study, carried out the study, performed the statistical analysis, and drafted the manuscript; $X W, Z L, C L$, JS, JS and TL: helped to carried out the study; XN: conceived and designed the study, edited and reviewed the manuscript. All authors read and approved the final manuscript.

\section{Funding}

This work is supported by General Program of Jiangsu Provincial Health Commission (No. M2020006), Changzhou Key Laboratory of Medical Physics (No. CM20193005), Changzhou Sci\&Tech program (No. CJ20200099), Young Talent Development Plan of Changzhou Health Commission (Nos. CZQM2020075 and CZQM2020067), the Science and Technology Programs for Young Talents of Changzhou Health Commission (No. QN201932).

\section{Availability of data and materials}

The datasets used during the current study are available from the corresponding author on reasonable request.

\section{Declarations}

\section{Ethics approval and consent to participate}

The study was approved by the ethics committee of Changzhou No.2 People's Hospital with the exemption for patient consent, because it is a retrospective study which would not bring extra risk to patient healthy and human rights (\#2020KY154-01).

\section{Consent for publication}

Not applicable.

\section{Competing interests}

The authors declare that they have no competing interests.

\section{Author details}

${ }^{1}$ Radiotherapy Department, Second People's Hospital of Changzhou, Nanjing Medical University, Changzhou 213003, China. ${ }^{2}$ Center for Medical Physics, Nanjing Medical University, Changzhou 213003, China. ${ }^{3}$ Oncology Department, Xuzhou No.1 People's Hospital, Xuzhou 221000, China. ${ }^{4}$ School of Biomedical Engineering and Informatics, Nanjing Medical University, Nanjing 213000, China.

Received: 24 March 2021 Accepted: 17 June 2021

Published online: 14 October 2021

\section{References}

1. Sorcini B, Tilikidis A. Clinical application of image-guided radiotherapy, IGRT (on the Varian OBI platform). Cancer/Radiother. 2006;10:252-7.

2. Wang $X, L i J$, Wang $P$, et al. Image guided radiation therapy boost in combination with high-dose-rate intracavitary brachytherapy for the treatment of cervical cancer. Brachytherapy. 2016;8:122-7.

3. Boda-Heggemann J, Lohr F, Wenz F, et al. kV cone-beam CT-based IGRT. Strahlenther Onkol. 2011;187:284-91.

4. Endo M, Tsunoo T, Nakamori $\mathrm{N}$, et al. Effect of scattered radiation on image noise in cone beam CT. Med Phys. 2001;28:469-74.
5. XuY Bai T Yan H, et al. A practical cone-beam CT scatter correction method with optimized Monte Carlo simulations for image-guided radiation therapy. Phys Med Biol. 2015:60:3567.

6. Kan MW, Leung LH, Wong W, et al. Radiation dose from cone beam computed tomography for image-guided radiation therapy. Int J Radiat Oncol Biol Phys. 2008;70(1):272-9.

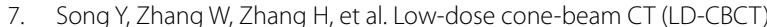
reconstruction for image-guided radiation therapy (IGRT) by threedimensional dual-dictionary learning. Radiat Oncol. 2020;15(1):192.

8. Rijcke BD, Geeteruyen RV, Rijcke ED, et al. Fast 3D CBCT imaging for lung SBRT: is image quality preserved? Radiother Oncol. 2017;123:S85-6.

9. Dunlop A, McQuaid D, Nill S, et al. Comparison of CT number calibration techniques for CBCT-based dose calculation. Strahlenther Onkol. 2015:191(12):970-8.

10. Giacometti V, King RB, Agnew CE, et al. An evaluation of techniques for dose calculation on cone beam computed tomography. Br J Radiol. 2019;92(1096):20180383.

11. Stankovic U, Ploeger LS, van Herk M, et al. Optimal combination of anti-scatter grids and software correction for CBCT imaging. Med Phys. 2017:44:4437-51.

12. Sisniega A, Zbijewski W, Badal A, et al. Monte Carlo study of the effects of system geometry and antiscatter grids on cone-beam CT scatter distribution. Med Phys. 2013;40:051915.

13. Sun M, Star-Lack JM. Improved scatter correction using adaptive scatter kernel superposition. Phys Med Biol. 2010;55:6695-720.

14. Kurz C, Kamp F, Park Y-K, et al. Investigating deformable image registration and scatter correction for CBCT-based dose calculation in adaptive IMPT: CBCT correction to enable IMPT dose calculation. Med Phys. 2016:43(10):5635-46.

15. Duan L, Ni X, Liu Q, et al. Unsupervised learning for deformable registration of thoracic CT and cone-beam CT based on multiscale features matching with spatially adaptive weighting. Med Phys. 2020;47:5632-47.

16. Arai K, Kadoya N, Kato T, et al. Feasibility of CBCT-based proton dose calculation using a histogram-matching algorithm in proton beam therapy. Phys Med. 2017;33:68.

17. Mainegra-Hing E, Kawrakow I. Fast Monte Carlo calculation of scatter corrections for CBCT images. J Phys Conf Ser. 2008;102:012017

18. Li J, Yao W, Xiao Y, et al. Feasibility of improving cone-beam CT number consistency using a scatter correction algorithm. J Appl Clin Med Phys. 2013;14(6):167-76.

19. Niu T, Sun M, Star-Lack J, et al. Shading correction for on-board conebeam CT in radiation therapy using planning MDCT images. Med Phys. 2010;37(10):5395-406.

20. Park YK, Sharp GC, Phillips J, et al. Proton dose calculation on scattercorrected CBCT image: Feasibility study for adaptive proton therapy. Med Phys. 2015;42(8):4449-59.

21. Li Y, Zhu J, Liu Z, et al. A preliminary study of using a deep convolution neural network to generate synthesized $C T$ images based on $C B C T$ for adaptive radiotherapy of nasopharyngeal carcinoma. Phys Med Biol. 2019;64(14):145010

22. Chen $L$, Liang $X$, Shen $C$, et al. Synthetic $C T$ generation from CBCT images via deep learning. Med Phys. 2020;47(3):1115-25.

23. Liang $X$, Chen L, Nguyen D, et al. Generating synthesized computed tomography (CT) from cone-beam computed tomography (CBCT) using CycleGAN for adaptive radiation therapy. Phys Med Biol. 2019;64(12):

24. Harms J, Lei Y, Wang T, et al. Paired cycleGAN based image correction for quantitative cone-beam CT. Med Phys. 2019;46(9):3998-4009.

25. Liu Y, Lei Y, Wang T, et al. CBCT-based synthetic CT generation using deepattention cycleGAN for pancreatic adaptive radiotherapy. Med Phys. 2020:47(6):2472-83

26. Kida S, Kaji S, Nawa K, et al. Visual enhancement of cone-beam CT by use of CycleGAN. Med Phys. 2020:47(3):998-1010.

27. Barateau A, De Crevoisier R, Largent A, et al. Comparison of CBCT-based dose calculation methods in head and neck cancer radiotherapy: from Hounsfield unit to density calibration curve to deep learning. Med Phys. 2020;47:4683-93.

28. Hansen DC, Landry G, Kamp F, et al. ScatterNet: a convolutional neural network for cone-beam CT intensity correction. Med Phys. 2018:45(11):4916-26. 
29. Lalonde A, Winey B, Verburg J, et al. Evaluation of CBCT scatter correction using deep convolutional neural networks for head and neck adaptive proton therapy. Phys Med Biol. 2020;65(24):245022.

30. Landry G, Hansen D, Kamp F, et al. Comparing Unet training with three different datasets to correct CBCT images for prostate radiotherapy dose calculations. Phys Med Biol. 2019;64(3):035011.

31. Thummerer A, Zaffino P, Meijers A, et al. Comparison of CBCT based synthetic CT methods suitable for proton dose calculations in adaptive proton therapy. Phys Med Biol. 2020;65(9):095002.

32. Ronneberger $\mathrm{O}$, Fischer P, Brox T, et al. U-Net: convolutional networks for biomedical image segmentation. In: International conference on medical image computing and computer-assisted intervention. 2015. p. 234-41.

33. Goodfellow I, Pougetabadie J, Mirza M, et al. Generative adversarial nets. Neural Inf Process Syst. 2014;27:2672-80.

34. Dong Y, Xu D, Zhou SK, et al, Automatic liver segmentation using an adversarial image-to-image network. In: International conference on medical image computing and computer assisted intervention (MICCAI). 2017. p. 507-15.

35. Rezaei M, Harmuth K, Gierke W, et al, A conditional adversarial network for semantic segmentation of brain tumor. In: International conference on medical image computing and computer assisted intervention. 2017. p. 241-52.

36. Madani A, Moradi M, Karargyris A, et al. Semi-supervised learning with generative adversarial networks for chest $x$-ray classification with ability of data domain adaptation. In: The IEEE international symposium on biomedical imaging. 2018. p. 1038-42.

37. McCollough $\mathrm{CH}$, Bartley AC, Carter RE, et al. Low-dose ct for the detection and classification of metastatic liver lesions. Med Phys. 2017;44(10):e339-52.

38. Maspero M, Savenije MHF, Dinkla AM, et al. Dose evaluation of fast synthetic-CT generation using a generative adversarial network for general pelvis MR-only radiotherapy. Phys Med Biol. 2018;63:185001.
39. Cusumano D, Lenkowicz J, Votta C, et al. A deep learning approach to generate synthetic CT in low field MR-guided adaptive radiotherapy for abdominal and pelvic cases. Radiother Oncol. 2020;153:205-12.

40. Quan TM, Nguyen-Duc T, Jeong WK. Compressed sensing MRI reconstruction using a generative adversarial network with a cyclic loss. IEEE Trans Med Imaging. 2018;37(6):1488-97.

41. Isola P, Zhu J, Zhou T, et al. Image-to-image translation with conditional adversarial networks. In: 2017 IEEE conference on computer vision and pattern recognition, CVPR 2017. p. 5967-76.

42. Zhu J, ParkT, Isola P, et al. Unpaired image-to-image translation using cycle-consistent adversarial networks. In: IEEE international conference on computer vision, ICCV 2017. p. 2242-51

43. Nie D, Trullo R, Lian J, et al. Medical image Synthesis with context aware generative adversarial networks. In: International conference on medical image computing and computer assisted intervention (MICCAI), 2017. vol. 10435. p. 417-25.

44. Tang Hao, Xu Dan, Sebe Nicu, et al, Attention-guided generative adversarial networks for unsupervised image-to-image translation. In: International joint conference on neural networks (IJCNN). 2019.

45. Bogaert $\mathrm{E}$, Monten C, Wagter CD, et al. Investigation of a fast CBCT protocol for supine accelerated whole breast. Irradiat Radiother Oncol. 2016;119:S434

46. Mao X, Li Q, Xie H, et al. Least squares generative adversarial networks. In: IEEE international conference on computer vision (ICCV), 2017.

47. Ramachandran P, Zoph B, Le QV. Searching for activation functions. 2017. arXiv:1710.05941.

\section{Publisher's Note}

Springer Nature remains neutral with regard to jurisdictional claims in published maps and institutional affiliations.
Ready to submit your research? Choose BMC and benefit from:

- fast, convenient online submission

- thorough peer review by experienced researchers in your field

- rapid publication on acceptance

- support for research data, including large and complex data types

- gold Open Access which fosters wider collaboration and increased citations

- maximum visibility for your research: over $100 \mathrm{M}$ website views per year

At BMC, research is always in progress.

Learn more biomedcentral.com/submissions 